\title{
Duodenal Gastrointestinal Stromal Tumor: A Rare Cause of Massive Upper GI Bleed managed with Segmental Resection of Duodenum
}

\author{
${ }^{1}$ Lohith Umapathi, ${ }^{2}$ Dinesh Bagaria, ${ }^{3}$ Vishal Attri, ${ }^{4}$ Lileswar Kaman
}

\begin{abstract}
Duodenal gastrointestinal stromal tumors (GIST) are relatively rare tumors and account for less than $5 \%$ of all cases of GIST, but still represents approximately $30 \%$ of primary duodenal tumors. Surgery is the only curative approach for duodenal GIST, but the optimal surgical procedure for duodenal GIST is not yet established. At present, there are no established guidelines regarding management of duodenal GIST. Various procedures including pancreaticoduodenectomy, pancreas-sparing duodenectomy, segmental duodenectomy, or wedge local resection have been described, but oncologic outcomes have not been evaluated. Buchs et al evaluated the role of segmental duodenal resection in the management of duodenal GIST and concluded that segmental resection is adequate for localized tumor where negative resection margins are obtainable. Here we present a case of duodenal GIST with massive upper GI bleed which was treated with segmental resection of the duodenum.
\end{abstract}

Keywords: GIST, Upper GI bleed, Duodenal resection.

How to cite this article: Umapathi L, Bagaria D, Attri V, Kaman L. Duodenal Gastrointestinal Stromal Tumor: A Rare Cause of Massive Upper GI Bleed managed with Segmental Resection of Duodenum. J Postgrad Med Edu Res 2014;48(3):148-150.

\section{Source of support: Nil}

Conflict of interest: None

\section{INTRODUCTION}

Duodenal gastrointestinal stromal tumors (GIST) account for less than $5 \%$ of all cases of GIST. ${ }^{1}$ Surgical resection is the curative treatment for duodenal GIST. ${ }^{2,3}$ Presently there are no established guidelines regarding surgical management of duodenal GIST. Various procedures including pancreaticoduodenectomy, pancreas-sparing duodenectomy, segmental duodenectomy, or wedge local resection have been described, but oncological outcomes have not been evaluated. Buchs et al evaluated the role of segmental duodenal resection in the management of duodenal GIST and

\footnotetext{
${ }^{1-3}$ Senior Resident, ${ }^{4}$ Additional Professor

${ }^{1-4}$ Department of General Surgery, Postgraduate Institute of Medical Education and Research, Chandigarh, India
}

Corresponding Author: Lileswar Kaman, Additional Professor Department of General Surgery, Postgraduate Institute of Medical Education and Research, Chandigarh, India, e-mail: kamanlil@yahoo.com concluded that segmental resection is adequate for localized tumor where negative resection margins are obtainable. ${ }^{3}$

\section{CASE REPORT}

A 51-year-old woman presented to our hospital with complaints of melena and hematemesis for 2 weeks with weakness and fatigue. She had undergone a cholecystectomy 2 years previously for symptomatic gall stone disease, but otherwise had no major problems in the past. She had hemoglobin of $5.8 \mathrm{gm} \%$, and normal liver and renal function tests. The coagulogram was normal. Upper GI endoscopy revealed a submucosal bulge at the junction of the second and third part of the duodenum with overlying telangiectatic blood vessels and evidence of fresh bleeding. Contrast enhanced computed tomography (CECT) abdomen showed a well-defined heterogeneously enhancing exophytic mass lesion of approximately $5.5 \times 4.9 \times 3.7 \mathrm{~cm}$ size arising from D3 segment of duodenum near D2-D3 junction with central nonenhancing area suggestive of necrosis (Fig. 1). There was no other evidence of metastatic disease. PET scan revealed FDG avid duodenal lesion of size $4.6 \times 4 \mathrm{~cm}$ in the D2-D3 area. Fat planes between the duodenal lesion and IVC was well maintained. The patient was subjected to surgery and underwent a segmental duodenectomy with end to end duodenoduodenostomy (Fig. 2). Postoperative

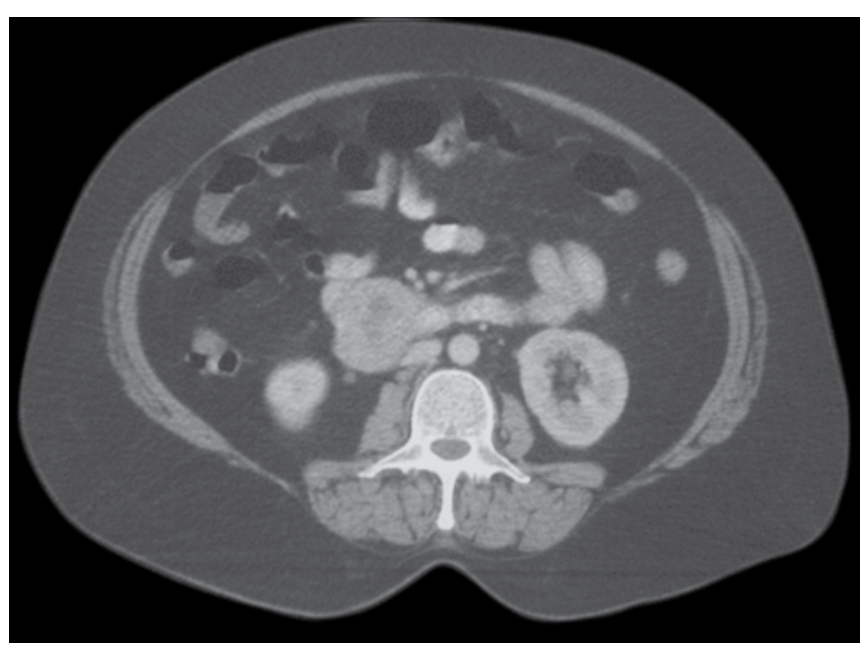

Fig. 1: CECT abdomen showing a well-defined heterogeneously enhancing exophytic mass lesion of approximately $5.5 \times 4.9 \times$ $3.7 \mathrm{~cm}$ size arising from D3 segment of duodenum near D2-D3 junction with central nonenhancing area suggestive of necrosis 


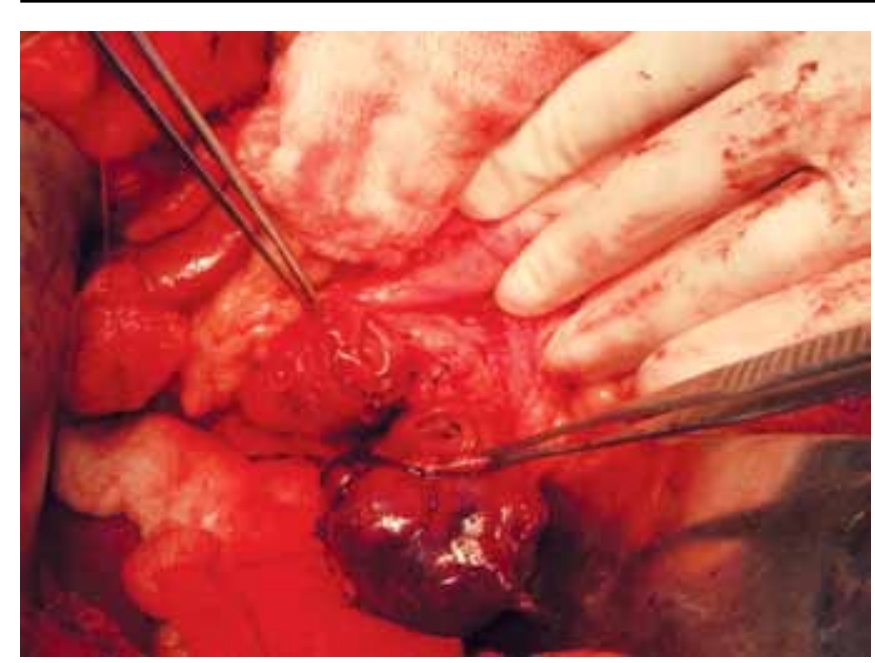

Fig. 2: Segmental duodenectomy with the tumor

recovery was unremarkable with complete resolution of symptoms. Histological examination of the lesion diagnosed a gastrointestinal stromal tumor (GIST) that was c-kit positive. Eighteen months later the patient is doing well with no evidence of disease recurrence or metastatic disease.

\section{DISCUSSION}

Gastrointestinal stromal tumors are usually low grade mesenchymal tumors of the gastrointestinal tract and are believed to originate from pluripotential mesenchymal stem cells designated to differentiate into interstitial cells of Cajal. ${ }^{1}$ Duodenal GIST are relatively rare tumor and account for less than $5 \%$ of all cases of GIST, but still represents approximately $30 \%$ of primary duodenal tumors. ${ }^{2,3}$ The term GIST was coined in 1983 when Mazur and Clark proposed that stromal tumors caused most mesenchymal tumors of the stomach and did not originate from smooth muscle. ${ }^{4}$ GIST show histological and immunochemical characteristics that are different from leiomyosarcomas, leiomyomas and schwannomas. Over the following years the term GIST came to be used for all mesenchymal tumors of the GI tract that do not arise from smooth muscle or Schwann cells. ${ }^{4,5}$

About $80 \%$ of GIST are characterized by the expression of a transmembrane receptor tyrosine kinase kit, a product of the c-kit proto-oncogene and identified by the expression of CD117. Around $5 \%$ of GIST harbor mutations in platelet derived growth factor receptor alpha (PDGFRA). ${ }^{6}$ Additionally, positivity for vimentin is seen in nearly all GIST and CD34 positivity is seen in 50 to $70 \%{ }^{7}$ Staining for smooth muscle actin may be positive in 30 to $40 \%$ while desmin (intermediate filament typical for muscle) and S-100 (a neural cell marker) are usually negative.

A high proportion of GIST arising from the stomach tends to be benign as compared to those from the small bowel, colon or esophagus; which tend to be more aggressive. GIST often present with GI bleed with no other symptoms. Patients with gastrointestinal hemorrhage are usually diagnosed by endoscopy, which may reveal a submucosal mass, but endoscopic biopsy has been shown to be diagnostic in only approximately $50 \%$ of cases. ${ }^{8,9}$ Historically, GIST has been associated with a 5-year survival of 48 to $65 \%$ following complete resection as against around 12 months in cases of incomplete resection. Recurrence is a common feature of high grade GIST seen in more than half the patients, which may be local recurrence or in the form of metastasis to the liver or rarely to regional lymph nodes. ${ }^{10,11}$ However, as unlike carcinomas, GIST do not widely infiltrate at the microscopic level and rarely metastasize to the lymph nodes. Therefore, local excision may be appropriate when technically feasible. In case of the duodenum, proximity and involvement of the ampulla of Vater warrants a Whipple's procedure, and a pancreas preserving resection of varying amounts of the duodenum may be done when feasible. Limited resection may be considered as a treatment option for duodenal GIST when technically feasible. Various limited resections for duodenal GIST have been reported depending on the site and the size of the tumors. Wedge resection with primary closure can be performed for small lesions. A segmental duodenectomy with side-to-end or end-to-end duodenojejunostomy may be performed for larger tumors located in the 3rd and 4th portions of the duodenum. A partial duodenectomy with Roux-en Y duodenojejunostomy is feasible for larger tumors involving the antimesenteric border of the 2 nd and 3 rd portions of the duodenum. ${ }^{12}$ In a series from the preimatinib era, Miettenen et al reporting on the outcome in 156 patients who had surgery for duodenal GIST, noted local recurrence, metastasis or both in $35 \%$ of their patients. ${ }^{13}$ Duodenal GIST vary widely in their aggressiveness from small indolent tumors to overt sarcomas. Duodenal GIST between 2 and $5 \mathrm{~cm}$ with a low mitotic rate have a low frequency of malignant behavior. Tumors which are large, with a high mitotic count, are reported to have a poor outcome and may not respond to imatinib mesylate. ${ }^{14,15}$ In general, a more favorable prognosis of duodenal GIST in comparison to GIST in other parts of the gastrointestinal tract is attributed to many factors, including the lower prevalence of p16 loss, lower K1-67L1 levels, smaller size of the lesion and lower mitotic count, duodenal location of the tumor and mutational status. ${ }^{14,15}$

Thus, duodenal GIST are a relatively uncommon subset of GIST which most commonly present with pain and upper gastrointestinal bleeding. Bleeding may require surgical palliation, though imatinib may also be used. Disease-free survival is often achieved by performing a curative surgical resection with a clear margin. Limited resection should be considered a treatment option for duodenal GIST when technically feasible. However, a Whipple's procedure would 
be required when a lesion involves the papilla or adjoining organs, such as the pancreas, or is large and has a high malignant potential. The risk of recurrence most often depends on tumor biology and aggressiveness. Complete resection with clear margins is the standard of care for all surgically resectable lesions in the duodenum.

\section{REFERENCES}

1. Miettinen M, Kopczynski J, Makhlouf HR, Sarlomo-Rikala M, Gyorffy H, Burke A, Sobin LH, Lasota J. Gastrointestinal stromal tumors, intramural leiomyomas, and leiomyosarcomas in the duodenum: a clinicopathologic, immunohistochemical, and molecular genetics study of 167 cases. Am J Surg Pathol 2003; 27(5):625-641.

2. Gold JS, Dematteo RP. Combined surgical and molecular therapy: the gastrointestinal stromal tumor model. Ann Surg 2006;244(2): 176-184.

3. Buchs NC, Bucher P, Gervaz P, Ostermann S, Pugin F, Morel P. Segmental duodenectomy for gastrointestinal stromal tumor of the duodenum. World J Gastroenterol 2010;16(22):27882792.

4. Mazur MT, Clark HB. Gastrointestinal stromal tumors: reappraisal of histogenesis. Am J Surg Pathol 1983;7(6):507-519.

5. Miettinen M, Lasota J. Gastrointestinal stromal tumor: definition, clinical, histological, immunohistochemical, and molecular genetic features and differential diagnosis. Virchows Arch 2001; 438(1):1-12.

6. Corless CL, Schroeder A, Griffich D, Town A, McGreevey L, Harrell P, Shiraga S, Bainbridge T, Morich J, Heinrich MC. PDGFRA mutations in gastrointestinal stromal tumours: frequency, spectrum and in vitro sensitivity to imatinib. J Clin Oncol 2005; 23(23):5357-5364.
7. Chung JC, Chu CW, Cho GS, Shin EJ, Lim CW, Kim HC, Song OP. Management and outcome of gastrointestinal stromal tumours of the duodenum. J Gastrointest Surg 2010;14(5):880-883.

8. Chak A, Canto MI, Rosch T, Dittler HJ, Hawes RH, Tio TL, LightdaleCJ, Boyce HW, Scheiman J, Carpenter SL, etal. Endosonographic differentiation of benign and malignant stromal cell tumors. Gastrointest Endosc 1997;45(6):468-473.

9. Conlon KC, Casper ES, Brennan MF. Primary gastrointestinal sarcomas: analysis of prognostic variables. Ann Surg Oncol 1995; 2(1):26-31.

10. Ng EH, Pollock RE, Munsell MF, Atkinson EN, Romsdahl MM. Prognostic factors influencing survival in gastrointestinal leiomyosarcomas. Implications for surgical management and staging. Ann Surg 1992;215(1):68-77.

11. McGrath PC, Neifeld JP, Lawrence W Jr, Kay S, Horsley JS, Parker GA. Gastrointestinal sarcomas. Analysis of prognostic factors. Ann Surg 1987;206(6):706-710.

12. Goh BK, Chow PK, Ong HS, Wong WK. Gastrointestinal stromal tumour involving the second and third portion of the duodenum: treatment by partial duodenectomy and Roux-en-Y duodenojejunostomy. J Surg Oncol 2005;91(4):273-275.

13. Fletcher CD, Bermar JJ, Corless C, Gorstein F, Lasota J, Longley BJ, Miettinen M, O’Leary TJ, Remotti H, Rubin BP, et al. Diagnosis of gastrointestinal stromal tumors: a consensus approach. Hum Pathol 2002;33(5):459-465.

14. Dematteo RP, Gold JS, Saran L, Gönen M, Liau KH, Maki RG, Singer S, Besmer P, Brennan MF, Antonescu CR. Tumor mitotic rate, size and location independently predict recurrence after resection of primary gastrointestinal stromal tumour (GIST). Cancer 2008;112(3):608-615.

15. Winfield RD, Hochwald SN, Vogel SB, Hemming AW, Liu C, Cance WG, Grobmyer SR. Presentation and management of gastrointestinal stromal tumors of the duodenum. Am Surg 2006; 72(8):719-722. 